\title{
GESTÃO EM ENFERMAGEM: REFLEXÕES ACERCA DOS DESAFIOS E ESTRATÉGIAS FRENTE À COVID-19
}

\section{NURSING MANAGEMENT: REFLECTIONS ON THE CHALLENGES AND STRATEGIES FACING COVID-19}

\author{
Ítalo Arão Pereira Ribeiro ${ }^{1} *$ Jefferson Abraão Caetano Lira ${ }^{2} *$ Sayonnara Ferreira Maia $^{3 *}$ \\ Rosângela Nunes Almeida ${ }^{4} *$ Márcia Astrês Fernandes ${ }^{5} *$ Lídya Tolstenko Nogueira $^{6} *$ Daniela Reis \\ Joaquim de Freitas ${ }^{7}$
}

\begin{abstract}
RESUMO
Objetivo: Refletir acerca dos desafios e das estratégias utilizadas pelo enfermeiro gestor frente à COVID-19. Metodologia: Trata-se de um estudo reflexivo temático realizado a partir de levantamento bibliográfico, no período de junho a julho de 2020, em bases eletrônicas de dados, por meio dos descritores controlados "Nursing", "Nursing, Team", "Coronavirus Infections", "Decision Making, Organizational", "Professional Competence" e "Strategies". $\mathrm{Na}$ análise, os dados foram organizados em categorias por similaridade de conteúdo. Resultados: Os principais desafios estão relacionados à escassez de recursos humanos, técnicos e de materiais, como a organização da assistência em Unidades de Terapia Intensiva improvisadas, a sobrecarga de trabalho, o estresse constante, o déficit de profissionais, a falta de treinamentos e de Equipamentos de Proteção Individuais, além das mudanças frequentes nos protocolos assistenciais. Dentre as estratégias gerenciais de enfermagem, destacam-se a reorganização de escalas, o uso de plataformas digitais, simulações, aplicação do Narsing Actives Score e das metodologias SBAR, 6S e ciclo PDCA. Considerações finais: Essas reflexões contribuem para despertar no enfermeiro gestor a necessidade de avançar na superação dos desafios e na apropriação de estratégias para o combate à COVID-19, no intuito de fortalecer o gerenciamento, a resolutividade e a integralidade do cuidado.

Palavras-chave: Competência Profissional. Tomada de Decisões Gerenciais. Gerenciamento da Prática Profissional. Infecções por Coronavírus. Enfermagem.
\end{abstract}

\begin{abstract}
Objective: To reflect on the challenges and strategies used by the nurse manager in front of COVID-19. Methodology: This is a reflective thematic study carried out from a bibliographic survey, from June to July 2020, in electronic databases, using the controlled descriptors "Nursing", "Nursing, Team", "Coronavirus Infections ", "Decision Making, Organizational ", "Professional Competence "and" Strategies ". In the analysis, the data were organized into categories by similarity of content. Results: The main challenges are related to the scarcity of human, technical and material resources, such as the organization of assistance in improvised Intensive Care Units, work overload, constant stress, deficit of professionals, lack of training and training. Personal Protective Equipment, in addition to frequent changes in care protocols. Among the nursing management strategies, the reorganization of scales, the use of digital platforms, simulations, application of the Narsing Actives Score and the SBAR, 6S and PDCA cycle methodologies stand out. Final considerations: These reflections contribute to awaken in the nurse manager the need to advance in overcoming challenges and in the appropriation of strategies to combat COVID-19, in order to strengthen management, resolvability and comprehensive care.
\end{abstract}

Keywords: Professional Competence. Decision Making, Organizational. Practice Management. Coronavirus Infections. Nursing.

\footnotetext{
${ }^{1}$ Enfermeiro. Doutorando em Enfermagem pelo Programa de Pós-Graduação em Enfermagem pela Universidade Federal do Piauí (PPGEnf/UFPI). Bolsista de mobilidade acadêmica pelo CNPq. Teresina, Piauí, Brasil. E-mail: italoaarao@ @otmail.com

${ }^{2}$ Enfermeiro. Doutorando em Enfermagem pelo Programa de Pós-Graduação em Enfermagem pela Universidade Federal do Piauí (PPGEnf/UFPI). Teresina, Piauí, Brasil. E-mail: j.abraaolira@gmail.com

${ }^{3}$ Enfermeira. Doutoranda em Enfermagem pelo Programa de Pós-Graduação em Enfermagem pela Universidade Federal do Piauí (PPGEnf/UFPI). Teresina, Piauí, Brasil. E-mail: sayonnaramaia1@gmail.com

${ }^{4}$ Enfermeira. Doutoranda pelo Programa de Pós-Graduação em Enfermagem pela Universidade Federal do Piauí (PPGEnf/UFPI). Teresina, Piauí, Brasil. E-mail: rnadasilva@ hotmail.com

${ }^{5}$ Enfermeira. Doutora em Ciências (Enfermagem Fundamental) pela Escola de Enfermagem de Ribeirão Preto da Universidade de São Paulo (EERP/USP). Professora Associada do Departamento de Enfermagem e do Programa de Pós-Graduação em Enfermagem da Universidade Federal do Piauí (PPGEnf/UFPI). Teresina, Piauí, Brasil. E-mail: m.astres@ ufpi.edu.br

${ }^{6}$ Enfermeira. Doutora em Enfermagem pela Escola de Enfermagem Anna Nery da Universidade Federal do Rio de Janeiro (EEAN/UFRJ). Professora Titular do Departamento de Enfermagem e do Programa de Pós-Graduação em Enfermagem da Universidade Federal do Piauí (PPGEnf/UFPI). Teresina, Piauí, Brasil. E-mail: lidyatn@ gmail.com

${ }^{7}$ Bióloga. Doutora em Ciências pela Universidade Federal do Rio Grande do Sul (UFRS). Professora Adjunta III no Departamento de Parasitologia e Microbiologia e do Programa de Pós-Graduação em Enfermagem da Universidade Federal do Piauí (PPGEnf/UFPI). Teresina, Piauí, Brasil. E-mail: danielarjfreitas@ufpi.edu.br
} 


\section{INTRODUÇÃO}

Com o surgimento do novo coronavírus, denominado cientificamente de Severe Acute Respiratory Syndrome Coronavirus (SARS-CoV-2), agente etiológico da Coronavirus Disease 2019 (COVID-19), e sua rápida disseminação global ${ }^{(1)}$, cresceu o número de pessoas infectadas a procura dos serviços de saúde, resultando no aumento dos índices de internações e de indivíduos necessitando de cuidados intensivos em serviços hospitalares. Tais fatos têm demandado do setor saúde a necessidade de recursos materiais e humanos para o enfrentamento desse agravo, que vem ocasionando profundas desordens e levado os sistemas de saúde pelo mundo ao colapso ${ }^{(2)}$.

A grande capacidade de transmissibilidade do novo coronavírus atinge não só a população geral, mas também muitos profissionais da saúde, em especial, a equipe de enfermagem, que, ao prestar cuidados diretamente aos pacientes e por lidar com a escassez de materiais e Equipamentos de Proteção Individuais (EPIs) ${ }^{(3)}$, torna-se uma das categorias mais vulneráveis aos riscos de infecção pela COVID-19, o que, por conseguinte, tem sido responsável por inúmeros afastamentos laborais, aumento da sobrecarga de trabalho, adoecimento mental e elevadas taxas de óbitos entre esses profissionais ${ }^{(4)}$.
Essas circunstâncias, além de afetarem diretamente a rotina laboral dos serviços de saúde, interferindo nas escalas e plantões dos profissionais, também influenciam negativamente na gestão de cuidados e na qualidade da assistência de enfermagem, contribuindo significativamente para intensificação da atual crise no setor da saúde. Nesse sentido, essa problemática requer do enfermeiro a necessidade do desenvolvimento de suas habilidades e competências gerenciais, que consistem em articular e integrar ações, favorecendo, qualificando e contextualizando a assistência, de forma previsível ou planejada ${ }^{(5)}$.

Nesse contexto, a prática e a gerência do cuidado em enfermagem são imprescindíveis para organização do trabalho e dos recursos humanos de enfermagem, pois sua atuação se baseia em competências gerais, em que estão inclusas a atenção à saúde, a tomada de decisões, a comunicação, a liderança, o gerenciamento, a educação permanente e competências específicas como: técnico-científicas, ético-políticas e socioeducativas (5-6). Essas características direcionam as atividades de enfermagem de forma mais coordenada e organizada para um cuidado qualificado, integral e eficiente ${ }^{(7)}$.

Dessa forma, as constantes mudanças, os avanços nos cenários das práticas assistenciais e a atual conjuntura da saúde, considerada pela World Health Organization (WHO) como uma das maiores crises 


\section{ARTIGO DE REFLEXÃO}

sanitárias já vistas no mundo, vêm repercutindo em mudanças na organização das equipes. Assim, esses aspectos exigem do enfermeiro conhecimento especializado, competências e habilidades gerenciais, como também experiência na área administrativa para organização dos setores, dos recursos materiais e equipamentos, para $\mathrm{O}$ recrutamento e dimensionamento de pessoal e do assistencialismo de enfermagem (3,8), principalmente, em meio a essas adversidades e potenciais situações que fragilizam a qualidade e resolutividade do cuidado em saúde.

Diante disso, este estudo tem como objetivo refletir acerca dos desafios e das estratégias utilizadas pelo enfermeiro gestor frente à COVID-19.

\section{METODOLOGIA}

Trata-se de um estudo reflexivo temático, desenvolvido durante a disciplina Bases Epistemológicas e Filosóficas das Ciências em Saúde e Enfermagem do curso de Doutorado em Enfermagem do Programa de Pós-Graduação em Enfermagem da Universidade Federal do Piauí (PPGEnf/UFPI). Esse tipo de estudo orienta o indivíduo, por meio da reflexão de fontes literárias, à compreensão e construção do conhecimento sobre um determinado assunto, aproximando-se da abordagem qualitativa, uma vez que interpreta e analisa elementos teóricos obtidos por meio do levantamento bibliográfico $^{(9-10)}$.

A investigação dos dados ocorreu no período de junho a julho de 2020, norteado pela busca de informações acerca dos desafios e das estratégias utilizadas pelo enfermeiro, mediante as competências gerenciais em tempos de pandemia da COVID-19. Utilizaram-se artigos científicos publicados em 2020, que contemplassem o objetivo proposto e estivessem disponíveis nas bases eletrônicas de dados: Cumulative Index to Nursing and Allied Health Literature (CINAHL); Medical Literature Analysis and Retrieval System Online (MEDLINE) via Pubmed e SCOPUS, empregando os descritores controlados MESH/CINAHL: "Nursing”, "Nursing, Team”, “Coronavirus Infections", "Decision Making, Organizational", "Professional Competence" e "Strategies". A delimitação temporal justifica-se pelo fato dessa pandemia ter iniciado em dezembro de 2019 e ainda está ocorrendo em várias regiões do planeta.

O processo de elegibilidade dos artigos, inicialmente, ocorreu pela leitura de títulos e resumos e, posteriormente, com a leitura na íntegra daqueles que se adequavam ao objetivo da reflexão. Nessa perspectiva, os artigos selecionados embasaram a construção dos resultados e discussão. Após leitura minuciosa e análise, foi possível organizar, por similaridade de conteúdo, a reflexão em dois núcleos de significância: desafios enfrentados pelo enfermeiro gestor frente à https://doi.org/10.31011/reaid-2021-v.95-n.33-art.1053 Rev Enferm Atual In Derme v. 95, n. 33, 2021 e-021044 
COVID-19; e estratégias no gerenciamento de enfermagem frente à COVID-19. Assim, foi possível discutir todas as informações à luz reflexiva da temática em questão.

\section{RESULTADOS E DISCUSSÃO}

\section{Desafios enfrentados pelo enfermeiro gestor frente à COVID-19}

A crise sanitária ocasionada pela COVID-19 trouxe inúmeros desafios tanto no âmbito assistencial quanto no aspecto administrativo. Assim, diversas competências gerenciais foram requeridas do enfermeiro em seu processo de trabalho, as quais são consideradas essenciais para que o serviço seja desenvolvido de forma efetiva, entretanto, considerando as competências individuais e organizacionais, vários desafios emergiram frente à COVID-19, como a carência de recursos humanos, técnicos e de materiais, que contribuíram para o agravamento da situação, dificultando a enfermeiro gestor.

No trabalho gerencial, os líderes da área de saúde devem tomar decisões baseadas em considerações científicas, econômicas, político-governamentais, éticas, socioculturais e organizacionais. Essas considerações influenciam os comportamentos de liderança, que afetam diretamente o trabalho e o bemestar da equipe de enfermagem da linha de frente ${ }^{(11)}$. funcionalidade dos serviços ofertados pelo

A utilização de Unidades de Terapia Intensiva (UTIs) improvisadas, a sobrecarga de trabalho dos profissionais de enfermagem, devido à redução do quadro de funcionários em detrimento do maior contágio da doença, e o estresse impactam diretamente nos serviços ofertados aos usuários e, consequentemente, no gerenciamento de enfermagem (12). Somando-se a isso outras dificuldades vão sendo incorporadas, como a falta de treinamentos para lidar com pacientes acometidos com a doença ${ }^{(13)}$.

A rotina de trabalho dos enfermeiros foi modificada, com a reorganização do cuidado ofertado aos pacientes com COVID19, para que menos intemperes aparecessem e que o trabalho fluísse de tal forma que não paralisasse o fluxo de assistência. Dessa maneira, os esforços dos gerentes de enfermagem em adequar a equipe sob sua responsabilidade, a complexidade da falta de EPIs, associada a sobrecarga emocional dos profissionais ao cuidar de pacientes infectados pela COVID-19, são grandes desafios ${ }^{(14)}$.

No Brasil, o Conselho Federal de Enfermagem já recebeu mais de 3,6 mil de denúncias sobre falta, escassez ou má qualidade de EPIs, incluindo máscaras, luvas e aventais, o que tem aumentado à preocupação tanto da população, quanto dos profissionais nos serviços de saúde, especialmente a equipe de enfermagem pela assistência contínua aos pacientes ${ }^{(13)}$.

A necessidade de uso de múltiplos EPIs pelos profissionais aumentou https://doi.org/10.31011/reaid-2021-v.95-n.33-art.1053 Rev Enferm Atual In Derme v. 95, n. 33, 2021 e-021044 
consideravelmente a carga de trabalho de enfermagem e a fadiga, pois o uso desses dispositivos de proteção eleva a temperatura corporal, sendo tolerável por poucas horas ${ }^{(15)}$ e, ainda, impossibilita o profissional de realizar necessidades básicas de nutrição e eliminação, além de ocasionarem lesão por pressão devido ao uso prolongado ${ }^{(13)}$.

Em face de tal situação, a reorganização e redução das jornadas de trabalho foram necessárias para diminuir o tempo de exposição e esgotamento, mas são dificultadas pela indisponibilidade de profissionais devido ao adoecimento ou por estarem nos grupos de maior risco. Assim, o recrutamento de novos profissionais de enfermagem apresentou-se imprescindível, no entanto trouxe consigo mais um problema para o enfermeiro gestor: o despreparo da maioria desses profissionais para atuarem nos setores mais requisitados, como UTIs e emergências.

Salienta-se que a reorganização dos serviços de saúde foi necessária não somente nas instituições de enfrentamento direto à COVID-19, como também nos serviços especializados que não puderam restringir seus atendimentos, como a assistência em hemodiálise (16) e à quimioterapia, os quais enfrentaram dificuldades semelhantes para a administração do cuidado.

Em outro aspecto, o medo público e a rápida disseminação da COVID-19 tornaram o planejamento e a organização dos serviços de saúde muito mais desafiadores para os https://doi.org/10.31011/reaid-2021-v.95-n.33-art.1053 enfermeiros gerentes, pois os profissionais de saúde comumente não tem formação e preparo para atenderem em pandemias ou desastres ${ }^{(18)}$. A pandemia, considerada um desastre natural, criou uma demanda sem precedentes por recursos humanos e materiais, que se esgotaram rapidamente.

Essa situação avassaladora fez surgir outro conflito administrativo, pois a habilidade gerencial de inspirar calma, confiança e respeito aos subordinados foi afetada na pandemia da COVID-19, diante de tantos dilemas e incertezas que repercutiram na gerência do cuidado ${ }^{(18)}$. Os profissionais de saúde esperam que a gerência e a instituição os orientem de forma correta, mas acabam experimentando dissonâncias cognitivas, diante de mudanças frequentes nas recomendações, à medida que surgem novas evidências cientificas, ocasionando mudanças constantes nos protocolos assistenciais ${ }^{(11)}$.

Estudo quantitativo com médicos e enfermeiros na Inglaterra evidenciou fragilidades na segurança profissional: somente um terço dos 850 entrevistados concordou que se sentia apoiado por confiança na gestão; apenas metade dos entrevistados relatou que foi fornecido treinamento adequado à equipe da linha de frente e dois terços dos entrevistados consideraram que não tinham EPIs disponíveis ${ }^{(19)}$.

Desse modo, o estresse psicológico vivenciado pelos profissionais de enfermagem possui causa multifatorial, como: trabalhar em Rev Enferm Atual In Derme v. 95, n. 33, 2021 e-021044 
um ambiente modificado e sobrecarrego; lidar com uma nova doença infecciosa; afastar-se da família; e o medo de estarem desprotegidos ${ }^{(20)}$. O adoecimento e mortes de pessoas à sua volta, incluindo colegas de trabalho, contribuiu para abalar a saúde mental dos profissionais ${ }^{(19)}$.

Muitos profissionais de saúde foram afastados dos serviços, devido a sua susceptibilidade à infecção. No Brasil, 3,4 \% deles morreram em decorrência da COVID19. Na Itália, $20 \%$ dos que trabalhavam na linha de frente tiveram a infecção e muitos morreram e, ainda, ao analisar dados dos profissionais atuantes no combate ao novo coronavírus, percebeu-se a presença de exaustão física e mental, dificuldades na tomada de decisão e maior risco de infecção desses ${ }^{(21)}$.

Os enfermeiros também estão sentindo medo do desconhecido e preocupação com o que está por vir, por si mesmos, por seus pacientes, colegas, bem como por suas próprias famílias e amigos. Além de enfermeiros, esses profissionais são pais, irmãos, amigos e parceiros com todas as preocupações compartilhadas pela maioria das pessoas, cuidando e protegendo de si, da família e dos pacientes ${ }^{(22)}$.

Outro desafio para o enfermeiro gestor é cuidar da saúde mental dos profissionais de enfermagem, pois esses sofrem com o estresse de cuidar dos doentes e de suas famílias ao mesmo tempo, durante a crise da COVID-19. Eles são especialmente vulneráveis ao https://doi.org/10.31011/reaid-2021-v.95-n.33-art.1053 Rev Enferm Atual In Derme v. 95, n. 33, 2021 e-021044 impacto psicológico dessa crise e correm riscos de desenvolverem depressão, ansiedade, insônia, estresse pós-traumático e esgotamento ${ }^{(11)}$. Nessa perspectiva, garantir a segurança dos profissionais de enfermagem é fundamental para minimizar esses impasses enfrentados pelos trabalhadores da linha de frente. Ressalta-se que guardar a confiança da equipe subordinada é um desafio importante aos enfermeiros gerentes para que mantenham sua liderança e autoridade, necessárias na administração do cuidado.

\section{Estratégias no gerenciamento de enfermagem frente à COVID-19}

Devido à intensa sobrecarga ocasionada pela COVID-19 aos serviços e profissionais da saúde, principalmente à equipe de enfermagem, UTI na Itália aplicou o Narsing Actives Score (NAS) para medir a carga de trabalho diária de enfermagem, a fim de gerenciar o cuidado, o qual indicou aumento de $33 \%$ da carga de trabalho durante a pandemia. Uma das mudanças na rotina, que impactou nessa sobrecarga, foi a implementação do posicionamento em prona em todos os pacientes com COVID-19 ${ }^{(15)}$. Assim, o NAS é uma ferramenta útil nesse contexto de pandemia, pois possibilita o dimensionamento adequado de enfermagem na terapia intensiva, o que contribui para a organização do processo de trabalho e para a segurança do paciente ${ }^{(23)}$. 
$\mathrm{Na}$ Espanha, as estratégias utilizadas no gerenciamento de enfermagem na UTI foram: a realocação de enfermeiros de outros setores que tinham experiência em cuidados críticos, a reorganização de escalas com seis horas de trabalho para reduzir o custo de EPIs e o risco de contágio, a suspensão de férias e folgas, aumento dos contratos de trabalho para período integral e a revisão de procedimentos para observar o grau de exposição e elencar os EPIs necessários ${ }^{(14)}$.

Ademais, os enfermeiros inexperientes em cuidados a pacientes críticos foram treinados por profissionais da própria unidade, enquanto fazia o trabalho diário. Ressalta-se que esses enfermeiros, inicialmente, ficavam apenas nas áreas limpas. Para minimizar a tensão desses profissionais, foi instituído o programa de treinamento Covid Critics Training 19, com Workshops presenciais, contendo simulações, e treinamentos online. Além disso, um guia dos medicamentos mais utilizados foi impresso, com o intuito de sanar dúvidas acerca da diluição, concentração, conservação e vias de administração. Essas práticas gerenciais foram imprescindíveis para a otimização dos cuidados de enfermagem ${ }^{(14)}$.

$\mathrm{Na}$ China, o gerenciamento da equipe foi pautado na comunicação eficiente, a partir da metodologia SBAR (situação, breve histórico, avaliação e recomendação), o que facilitou a passagem de plantão, reduzindo as omissões e as chances de erros. Para minimizar a contaminação dos profissionais e https://doi.org/10.31011/reaid-2021-v.95-n.33-art.1053 garantir de segurança organizacional, empregou-se a metodologia 6S, que consiste na utilização, arrumação, limpeza, saúde e higiene, organização e autodisciplina. Além do mais, passaram a monitorar os profissionais em relação aos sinais e sintomas gripais ${ }^{(20)}$. Destaca-se que essas metodologias de gestão, que infelizmente não é a realidade em muitos cenários brasileiros, permitem a integralidade do cuidado e a segurança dos pacientes e dos profissionais.

A liderança, exercida pelo gerente de enfermagem, com informações claras e precisas, bem como a preocupação com as condições de trabalho da equipe e a busca incessante por melhorias, é fundamental para passar credibilidade e motivar a equipe de enfermagem. As mentorias realizadas por enfermeiros experientes, a partir de mídias digitais e redes sociais, também são ferramentas estratégicas que possibilitaram trocas de experiências e direcionamentos, principalmente para aqueles profissionais que não contaram com treinamentos e educação continuada no enfrentamento da COVID-19 (11). Isso reforça a importância de gestores capacitados e com expertise na área, além da reinvenção da enfermagem na difusão do conhecimento.

O uso das plataformas digitais facilita a tomada de decisões pela equipe interdisciplinar, otimizando a assistência às pessoas com COVID-19 e a gestão dos serviços de saúde. Essas plataformas também Rev Enferm Atual In Derme v. 95, n. 33, 2021 e-021044 


\section{ARTIGO DE REFLEXÃO}

garantiram a humanização da assistência, uma vez que possibilitaram a comunicação entre pacientes internados e familiares ${ }^{(24)}$. Além do mais, o enfermeiro, no gerenciamento do cuidado, pode utilizar a publicidade da mídia para promover educação em saúde e conscientizar às pessoas a respeito das formas de prevenção, controle e dos riscos da COVID-19 ${ }^{(25)}$. Afinal, vivemos na era das fake news e as informações corretas precisam chegar à população.

A preocupação com a saúde mental dos profissionais de enfermagem foi evidenciada na Nova Zelândia, onde os profissionais foram inseridos em um grupo de bem-estar, além de contarem com o uso de um aplicativo móvel e assistência psicológica, que ofereceram apoio, atenção e carinho a cada um, o que amenizou essa rotina de incertezas, medo e ansiedade frente à COVID-19 ${ }^{(24)}$. Desse modo, iniciativas como essa devem ser expandidas mundialmente, pois os gestores e profissionais de enfermagem da linha de frente, devido à rotina estressante e ao risco elevado de infecção pelo novo coronavírus, acabam apresentando esgotamento físico e emocional e, muitas vezes, sentem-se desamparados.

A aplicação do ciclo PDCA (planejar, fazer, checar e agir) foi uma estratégia de gerenciamento empregada para padronizar e melhorar a qualidade e o desempenho da equipe de enfermagem na China. No planejamento, foram levantados os problemas e possíveis soluções. Dentre as problemáticas https://doi.org/10.31011/reaid-2021-v.95-n.33-art.1053 evidenciadas na UTI COVID-19, destacaramse: áreas contaminadas não claramente definidas, papéis e responsabilidades pouco claros, desorganização de insumos, transferência ineficaz de plantão e tempo elevado de espera para recepção de medicações ${ }^{(26)}$.

Assim, após as sugestões da equipe, foi realizado o plano gerencial, o qual contemplou todas as demandas identificadas. $\mathrm{Na}$ etapa do fazer, foram desenvolvidas capacitações e atualizações regulares com foco nos problemas. Na checagem, o gerente de enfermagem fazia inspeção, no mínimo semanal, para avaliar o desempenho da equipe, utilizando formulário. $\mathrm{Na}$ etapa do agir, foram oferecidas melhorias e identificados novos problemas para o próximo ciclo. Observou-se que o ciclo PDCA melhorou o profissionalismo e as habilidades da equipe de enfermagem, garantindo a qualidade da assistência, sobressaindo-se como uma ferramenta de gerenciamento eficaz no contexto da COVID-19 (26).

Por fim, assim como os demais tipos de pesquisa, essa reflexão apresentou limitações originadas pela ausência de estudos mais específicos e voltados para temática. A falta de pesquisas com metodologias mais robustas e de caráter investigativo, apresentaram-se como as principais restrições encontradas para uma discussão mais aprofundada sobre o assunto. 


\section{CONSIDERAÇÕES FINAIS}

Nessa perspectiva, enfatiza-se que os principais desafios enfrentados pelo enfermeiro gestor frente à COVID-19 estão relacionados à escassez de recursos humanos, técnicos e de materiais. Dessa forma, a organização da assistência em UTIs improvisadas, a sobrecarga de trabalho, o estresse constante, o déficit de profissionais, a falta de treinamentos e de EPIs, além das mudanças frequentes nos protocolos assistenciais são entraves vivenciados no gerenciamento de enfermagem.

Esse cenário desafiador de pandemia da COVID-19 mostrou o protagonismo da enfermagem na assistência, bem como a capacidade gerencial do enfermeiro, com o desenvolvimento de ações estratégicas, como a reorganização de escalas, o uso de plataformas digitais, treinamentos com simulações clínicas, aplicação do NAS e das metodologias SBAR, 6S e ciclo PDCA, que impulsionam e fortalecem a resolutividade do cuidado, a integralidade, a qualidade e a segurança na assistência.

Portanto, essas reflexões contribuem para despertar no enfermeiro gestor a necessidade de avançar na superação dos desafios e na apropriação de estratégias para o combate à COVID-19. Nesse pressuposto, sugere-se a realização de estudos originais acerca da temática, a fim de nortear ações mais assertivas no gerenciamento de enfermagem.

\section{REFERÊNCIAS}

1. Meneses AS. Gerenciamento Emergencial de Recursos da Atenção Primária a Saúde no Enfrentamento à Pandemia da COVID-19. SciELO Preprint. 2020;1(1):1-13. Disponível em:

https://doi.org/10.1590/SciELOPrepri nts.557

2. Miranda FMDA, Santana L, Pizzolato AC, Sarquis LMM. Working conditions and the impact on the health of the nursing professionals in the context of covid-19. Cogitare enferm. 2020; (25):e72702. Disponível em: http://dx.doi.org/10.5380/ce.v25i0.727 $\underline{02}$

3. Pereira MD, Torres EC, Pereira MD, Antunes PFS, Costa CFT. Sofrimento emocional dos Enfermeiros no contexto hospitalar frente à pandemia de Covid-19. Research, Society and Development. 2020; 9(8):e67985121. Disponível em: http://dx.doi.org/10.33448/rsdv9i8.5121

4. Souza SLPS, Souza AG. Enfermagem brasileira na linha de frente contra o novo Coronavírus: quem cuidará de quem cuida? J nurs health. 2020; 10(n.esp.): e20104005. Disponível em: 
https://periodicos.ufpel.edu.br/ojs2/ind ex.php/enfermagem/article/view/1844 $\underline{4 / 11237}$

5. Treviso P, Peres SC, Silva AD, Santos AA. Competências do enfermeiro da gestão do cuidado. Rev Adm Saúde. 2017; 7(69):14. Disponível em: http://dx.doi.org/10.23973/ras.69.59

6. Souza JO, Machado VB, Sousa ALRS. Competências gerenciais do enfermeiro: uma revisão integrativa. Rev Ciências da Saúde e Educação IESGO. 2019; 1(2):1-20. Disponível em:

http://revista.iesgo.edu.br/ojs/index.ph $\mathrm{p} / \mathrm{CSEI} / \mathrm{article} / \mathrm{view} / 27$

7. Berghetti L, Franciscatto LHG, Getelina CO. Formação do Enfermeiro Acerca do Gerenciamento: Entraves e Perspectivas. Rev enferm Centr-Oeste Min. 2019; 9:e2820. Disponível em: http://dx.doi.org/10.19175/recom.v9i0. $\underline{2820}$

8. Soares MI, Resck ZMR, Terra FS. Saberes gerenciais do enfermeiro no contexto hospitalar. Rev bras enferm. 2016; 69(4):676-83. Disponível em: http://dx.doi.org/10.1590/0034-

\subsection{9i}

9. Silva JS, Carvalho ARB, Leite HDCS, Oliveira EMN. Reflexiones sobre los riesgos ocupacionales en trabajadores de salud en tiempos pandémicos por COVID-19. Rev cuba enferm. 2020; 36(2):e3738. Disponível em: http://www.revenfermeria.sld.cu/index

.php/enf/article/view/3738

10. Ibiapina ARS, Monteiro CFS, Silva Júnior FJG, Costa APC, Campos LRB, Brito VS. Oficinas terapêuticas em Centro de Atenção Psicossocial: para além dos muros da loucura. Rev enferm UFPI. 2019; 8(3):92-5. Disponível em: https://doi.org/10.26694/22387234.8392-95

11. Caresse L, Connie V. A pandemic crisis: mentoring, leadership, and the millennial nurse. Nursing Economic. 2020; 38(3):152-4. Disponível em: http://www.nursingeconomics.net/necf iles/2020/MJ20/152.pdf

12. Raurell-Torredà M, Martínez-Estalella G, Frade-Mera MJ, Carrasco RRLF, Romero ESP._Reflexiones derivadas de la pandemia COVID-19. Enfermería Intensiva. 2020; 31(2):903 .

Disponível

em:

https://doi.org/10.1016/j.enfi.2020.03. $\underline{002}$

13. Oliveira AC. Desafios da enfermagem frente ao enfrentamento da pandemia da Covid19. REME rev min enferm. 2020; 24:e-1302. Disponível em: http://www.dx.doi.org/10.5935/14152762.20200032

14. Raurell-Torredà M. Gestión de los equipos de enfermeira de UCI durante la pandemia Covid-19. Enferm intensiva. 2020; 31(2):49-51. 
Disponível

em:

https://doi.org/10.1016/j.en

fi.2020.04.001

15. Lucchini A, Giani M, Elli S, Villa S, Rona R, Foti G. Nursing Activities Score is increased in Covid-19 patients. Intensive Criti Care Nurs. 2020; 59(1):102876. Disponível em: https://doi.org/10.1016/j.iccn.2020.10 $\underline{2876}$

16. Harwood L. Pandemic Uncertainty: Considerations for Nephrology Nurses. Nephrol Nurs J. 2020; 47(2):127-30.

https://doi.org/10.37526/1526744X.2020.47.2. 127

17. Ramos RS. A Enfermagem Oncológica no Enfrentamento da Pandemia de Covid-19: Reflexões e Recomendações para a Prática de Cuidado em Oncologia. Rev bras cancerol. 2020; 66(TemaAtual):1-5. Disponível em: https://rbc.inca.gov.br/revista/index.ph p/revista/article/view/1007

18. Middaugh DJ. Maintaining Management During Disaster: The Covid-19 Edition. Nursi manage. 2020; 29(3):211-2. Disponível em: http://search.ebscohost.com/login.aspx ?direct $=$ true $\&$ profile $=$ ehost $\&$ scope $=$ sit e\&authtype $=$ crawler $\& j \mathrm{rnl}=10920811$ $\underline{\& A N=143616920 \& h=300 A F \% 2 B C}$ XvCx3I3Gu6nHAsyPlnZurG65IBRdF 9RnMoCKhPqBHNn2fco47dsc0A5a https://doi.org/10.31011/reaid-2021-v.95-n.33-art.1053
mnnMEmtKY7RDOdvGeU4G10g\%3

$\underline{\mathrm{D} \% 3 \mathrm{D} \& \mathrm{crl}=\mathrm{f}}$

19. Iqbal MR, Chaudhuri A. COVID-19: Results of a national survey of United Kingdom healthcare professionals' perceptions of current management strategy - A cross-sectional questionnaire study. Int J Surg. Disponível em: https://doi.org/10.1016/j.ijsu.2020.05. $\underline{042}$

20. Tang L, Zhao XM, Yu XY. Team management in critical care units for patients with COVID-19: an experience from Hunan Province, China. Crit care. 2020; 24(304)1-3. https://doi.org/10.1186/s13054-02002921-7

21. Medeiros EA. A luta dos profissionais de saúde no enfrentamento da COVID-19. Acta Paul Enferm. 2020;33:e-EDT20200003. Disponível em: $\quad$ https://doi.org/10.37689/actaape/2020edt0003

22. Jackson D, Bradbury-Jones C, Baptiste D, Gelling L, Morin K, Neville $S$, et al. Life in the pandemic: Some reflections on nursing in the context of COVID-19. J Clin Nurs. 2020; 29(1):2041-43. Disponível em: https://doi.org/10.1111/jocn.15257

23. Cyrino CMS, Dell'Acqua MCQ, Castro MCN, Oliveira EM, Deodato S, Almeida PMV. Nursing Activities Score by assistance sites in Intensive Rev Enferm Atual In Derme v. 95, n. 33, 2021 e-021044 
Care Units. Esc Anna Nery Rev Enferm. 2018; 22(1):e20170145. https://www.scielo.br/pdf/ean/v22n1/p t 1414-8145-ean-2177-9465-EAN2017-0145.pdf

24. Sutton-Smith L. Planning for a Covid19 crisis: a new era of critical care nursing began at Wellington Hospital's intensive care unit in March as staff began preparing for a possible catastrophe. Nurs N Z. 2020; 26(4):26-28.

25. Zhang XJ, Shi TY, Sun L. COVID-19: What is next for nursing in public health emergency? Nurse educ pract. 2020; 46:102821. Disponível em: https://doi.org/10.1016/j.nepr.2020.10 $\underline{2821}$

26. Chen Y, Zheng J, Wu D, Zhang Y, Lin Y. Application of the PDCA cycle for standardized nursing management in a COVID-19 intensive care unit. Ann palliat med. 2020; 9(3):1198-205.

Disponível em: http://dx.doi.org/10.21037/apm-201084

Submissão: 2021-03-15

Aprovado: 2021-03-19 